\title{
Translational Reviews
}

\section{Mucin Granule Intraluminal Organization}

\author{
Juan Perez-Vilar \\ Cystic Fibrosis/Pulmonary Research and Treatment Center, School of Medicine, University of North Carolina at Chapel Hill, Chapel Hill, \\ North Carolina
}

\begin{abstract}
Mucus secretions have played a central role in the evolution of multicellular organisms, enabling adaptation to widely differing environments. In vertebrates, mucus covers and protects the epithelial cells in the respiratory, gastrointestinal, urogenital, visual, and auditory systems, amphibian's epidermis, and the gills in fishes. Deregulation of mucus production and/or composition has important consequences for human health. For example, mucus obstruction of small airways is observed in chronic airway diseases, including chronic obstructive pulmonary disease, asthma, and cystic fibrosis. The major protein component in the mucus is a family of large, disulfide-bonded glycoproteins known as gel-forming mucins. These proteins are accumulated in large, regulated secretory granules (the mucin granules) that occupy most of the apical cytoplasm of specialized cells known as mucous/goblet cells. Since mucin oligomers have contour dimensions larger than the mucin granule average diameter, the question arises how these highly hydrophilic macromolecules are organized within these organelles. I review here the intraluminal organization of the mucin granule in view of our knowledge on the structure, biosynthesis, and biophysical properties of gel-forming mucins, and novel imaging studies in living mucous/goblet cells. The emerging concept is that the mucin granule lumen comprises a partially condensed matrix meshwork embedded in a fluid phase where proteins slowly diffuse.
\end{abstract}

Keywords: granule matrix; mucin granules; mucins; secretory granules; secretion

\section{GEL-FORMING MUCINS: STRUCTURE AND BIOSYNTHESIS}

Five gel-forming mucins (MUC2, MUC5AC, MUC5B, MUC6, and MUC19) have been found in humans. These mucins share a similar structural organization (Figure 1A) characterized by $(1,2)$ : $(i)$ multi-domain polypeptide chains with thousands of amino acid residues; (ii) a large, centrally located region mainly consisting of threonine and/or serine-rich tandemly repeated sequences to which O-linked oligosaccharides are covalently bound; (iii) the presence of several under-glycosylated, Cys-rich domains that are conserved among different mucins; (iv) the formation of disulfide-linked species ranging from dimers to more than 16 oligomers (3); and ( $v)$ molecular polydispersity because of genetic polymorphism (e.g., multiple alleles encoding

(Received in original form August 9, 2006 and in final form August 22, 2006)

The research in the author's laboratory was supported by the Cystic Fibrosis Foundation (grants PEREZV04G0 and PEREZV06G0) and the National Institutes of Health-NIDDK (grant DK67404).

Correspondence and requests for reprints should be addressed to Juan Perez-Vilar, Cystic Fibrosis/Pulmonary Research and Treatment Center, School of Medicine, University of North Carolina at Chapel Hill, CB\#7248, Chapel Hill, NC 275997248. E-mail: juan_vilar@med.unc.edu

Am J Respir Cell Mol Biol Vol 36. pp 183-190, 2007

Originally Published in Press as DOI: 10.1165/rcmb.2006-0291TR on September 7, 2006

Internet address: www.atsjournals.org variable number of tandem repeats) (4), diverse O-glycosylation pattern, proteolytic processing, and/or different degree of oligomerization.

Mucin monomers are synthesized in the endoplasmic reticulum, where they are $\mathrm{N}$-glycosylated and likely $\mathrm{C}$-mannosylated, and form disulfide-linked dimers $(1,5)$ (Figure 1B). Once in the Golgi complex, dimeric mucin precursors are O-glycosylated, sulfated, and assembled into disulfide-bonded oligomers/ multimers by interdimeric disulfide bonds $(1,6)$. Proteolytic processing may occur as well in these late secretory compartments (7). Fully processed mucins are secreted by constitutive and $\mathrm{Ca}^{2+}$-dependent regulated mechanisms that are not fully understood $(8,9)$. Mucins secreted by the regulated pathway are accumulated in the mucin granules of mucous/goblet cells $(7,10)$ until proper regulatory signals activate their exocytosis. In contrast to other regulated secretory vesicles/granules, the major secretory product in the mucin granule is also the key component of the intragranular matrix, as attested by the absence of mucin granules in intestinal "mucous" cells of mice lacking expression of $m M u c 2$, the main gel-forming mucin gene expressed in the intestine (11). Hence, the mechanisms of intragranular mucin sorting and packaging (i.e., matrix formation) must be synchronized or simply function as a single mechanism during mucin granule biogenesis.

\section{GEL-FORMING MUCINS: TOPOLOGICAL ASPECTS}

Biophysical data obtained with extracellular mucus/mucins, together with some basic principles of polymer physics, provide us with a rational view on the magnitude of the topological problems associated with the large sizes of mucin macromolecules. Based on the available evidence, we can assume that a typical gel-forming mucin (e.g., MUC5AC): $(i)$ has $\sim 5,000$ amino acid residues per monomer; $(i i)$ is assembled into disulfidelinked, linear oligomers with 16 or more monomers each, and contour lengths $(L) \geqslant 8 \mu \mathrm{m}$; and (iii) forms flexible oligomeric random coils with a (cylindrical) Kuhn or effective segmentthat is, the minimal polypeptide segment that can be considered rigid (12, 13), of length $l \sim 30 \mathrm{~nm}$ and diameter $d \sim 7 \mathrm{~nm}$ (14-16).

Because mucins are negatively charged, highly hydrophilic macromolecules, mucin random coils can be considered as selfavoiding (i.e., the chains cannot cross one another because of interchain repulsion) linear polymers $(12,13)$. In a diluted solution, the radius of gyration $\left(R_{g}\right)$, defined as the average distance from all Kuhn segments to the center of mass of the oligomer $(12,13)$, and the pervade volume $(V)$, defined as the spherical volume of radius $R_{g}$ of each oligomeric chain, can be approximated by the following expressions:

$$
R_{g} \sim \frac{l^{4 / 5} \times d^{1 / 5} \times N^{0.588}}{2.45} \text { and } V \sim 4 \times \frac{\pi}{3} \times R_{g}^{3}
$$




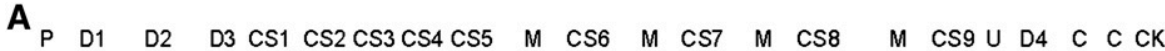

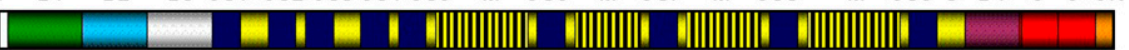

B

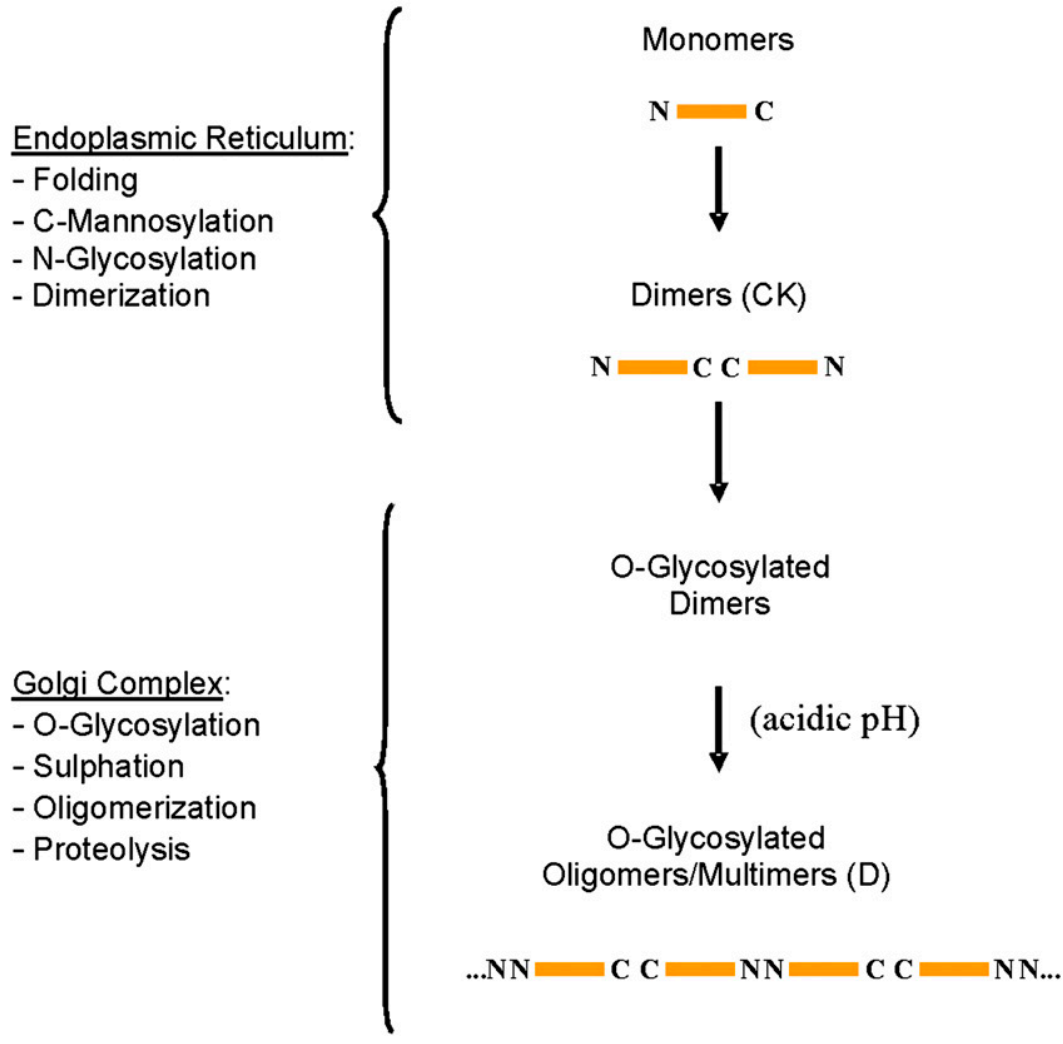

Figure 1. Structure and biosynthesis of gelforming mucins. $(A)$ Schematic representation of the structure of MUC5AC $(>5,000$ amino acid residues), a typical gel-forming mucin, showing the major protein domains found in its structure, as deduced from cDNA and genomic sequencing (e.g., Refs. 58, 59). P, signal peptide; U, Ser/ Thr-rich unique sequences; $M$, tandemly repeated Ser/Thr-rich sequences-containing domains; D, D-domains; CS, Cys subdomains; C, C-domains; CK, CK (cystine-knot)-like domain. The $\mathrm{U}$ and $\mathrm{M}$ domains are heavily O-glycosylated. D-, CS-, C-, and CK-like domains are underglycosylated and Cys-rich protein domains. (B) Current model for the biosynthesis and covalent assembly of gel-forming mucins. Note that the covalent assembly of mucins involves the formation of inter-chain disulfide bonds between the C-terminal CK-domain in two monomers while in the endoplasmic reticulum, and inter-chain disulfide bonds connecting the $\mathrm{N}$-terminal D-domains in dimers to form oligomers of variable size.
Where $N$ is the total number of Kuhn segments (i.e., $\sim L / l$ ). For our 16-mer mucin oligomer, the corresponding $R_{g}$ and $V$ values would be $\sim 0.24 \mu \mathrm{m}$ and $\sim 0.05 \mu \mathrm{m}^{3}$, respectively, which are in the same range of the hydrodynamic sizes determined empirically (14-17).

Above a certain concentration, known as the threshold concentration or $c^{*}$, self-avoiding linear polymeric chains begin to overlap and eventually form a heavily entangled network (12, 13). The dimensionless counterpart of $c^{*}$, the volume fraction $\left(\phi^{*}\right)$ (i.e., the volume occupied by the polymer chains in the solution of volume $V$ ), can be obtained from the expression:

$$
\phi^{*} \sim \frac{L}{V} \times d^{2}
$$

Thus, for the 16 -mer mucin oligomer, $\phi^{*} \sim 0.008$, which means that strand overlapping starts when just $0.8 \%$ of the available volume is filled by the mucin oligomers. Hence, due to their large sizes mucin oligomers begin to overlap and form entanglements when they occupy a very small fraction of the space in which they are confined. Indeed, both native and purified mucins are well known to form entangled networks (e.g., Refs. 14, 17), although several studies also point to the existence of noncovalent physical cross-links among mucin oligomers in the mucus (e.g., Refs. 15, 16, 18). It is possible that the contribution of noncovalent interactions is more significant in certain environments, for example, the stomach lumen $(15,16)$, or specific intracellular compartments (see below). Importantly, the movement of single polymer chains inside a heavily entangled network occurs by a reptation-like mechanism in which chain slow diffusion is inversely related to its length $(12,13,19)$. Therefore, shorter mucin oligomers diffuse faster and ultimately their solutions are less viscous and more dispersible than those with larger mucin oligomers, as attested by the decrease in viscosity of mucus or mucin solutions incubated with disulfide bond-reducing agents and/or proteolytic enzymes (20).

In concentrated polymer solutions, the distance between monomers in adjacent strands can be assessed by determining the correlation length $(\xi)(12,13)$. For a linear polymer with cylindrical Kuhn segments, $\xi$ can be approximated by the following expression:

$$
\xi \sim \phi^{-0.76} \times l \times\left(\frac{l}{d}\right)^{0.23}
$$

For our test gel-forming mucin, $\xi$ is $\geqslant 100 \mathrm{~nm}$ only when $\phi \leqslant 0.32$, that is, when the volume occupied by the mucin oligomers is $\leqslant 32 \%\left(\phi>>\phi^{*}\right)$ of the available space. It can be concluded that: $(i)$ formation of a dense tangled network does not necessarily mean the disappearance of inter-strand space (i.e., the network pores); and (ii) the higher the flexibility of the mucin (i.e., the smaller the Kuhn segment), the lesser the interoligomer distance.

\section{GEL-FORMING MUCINS: STRUCTURAL CHANGES DURING BIOSYNTHESIS AND INTRACELLULAR TRAFFICKING}

Irrespective of the nature of the inter-oligomer links, mucins in mucus gels form a three-dimensional network in which relatively large proteins can diffuse through their pores (14-16). Although the organization of intracellular mucins is virtually unknown, a 
reasonable picture can be proposed in view of the information reviewed in the preceding sections, results from early biochemical investigations, and recent live cell imaging investigations (see next section below). Studies with purified, de-glycosylated native mucins support the view that in the endoplasmic reticulum the largely uncharged and non-O-glycosylated mucin precursors (monomers and dimers) likely form globular but highly flexible random coils $(16,21,22)$, which is consistent with the fact that the large central domains comprise repetitive sequences rich in Ser and Thr, and often Gly and Pro residues. Highly flexible chains can form more compact structures and consequently facilitate the transport of mucin precursors out of the endoplasmic reticulum.

Once mucin precursors reach the proximal compartments of the Golgi complex, the addition of GalNAc residues to the mucin Ser/Thr-rich central regions limits the rotation of the peptide bonds implicated; leading to less flexible strands (21). This property explains the relatively large Kuhn segment $(l)$ of glycosylated mucins (15). Hence, initiation of O-glycosylation will reduce chain flexibility while increasing the inter-oligomer space, as $\xi$ is directly proportional to $l$. The intralumenal concentration of mucins in the endoplasmic reticulum or the Golgi complex has not been yet determined but it is expected to be high in view of microscopic studies (e.g., 23). Thus, similarly to mucin oligomers, mucin dimers probably overlap at a very low intralumenal concentration ( $\phi^{*} \geqslant 3 \%$; see above), and hence formation of entanglements could begin early during mucin biosynthesis. However, the fact that mucin precursors are extensively and efficiently O-glycosylated while rapidly traversing the Golgi complex (1) suggests that at these locations, the mucin precursors form a dynamic and penetrable network that does not impede glycosylation. Continuous reptation of mucin precursors in the network likely makes possible their post-translational modification.

Important changes occur in the distal (trans-) Golgi compartments. First, intraluminal $\left[\mathrm{H}^{+}\right]$and $\left[\mathrm{Ca}^{2+}\right]$ increase $(24,25)$, acidifying the lumen and increasing its ionic strength. Second, mucin dimers are assembled into multimers by inter-dimeric disulfide bonds (Figure 1B). The synthesis of larger macromolecules means decreased $\phi^{*}$ and a significant increase in luminal viscosity, as this property is directly proportional to $N^{3}(12,13)$. Third, extensive sulfation and sialylation of $\mathrm{N}$ - and O-linked oligosaccharide chains in mucins (1), and eventually the conversion of mucins to polyanionic macromolecules, occurs. High negative charge density contributes to the stiffness of the mucin oligomers by increasing the repulsion between adjacent negatively charged rests $(21,22)$. Altogether, these changes create appropriate conditions for novel interchain interactions beyond topological entanglements.

First, $\mathrm{Ca}^{2+}$-mediated interchain ionic interactions linking the sialic and sulfate residues in mucins are then possible $(14,26)$. These interactions could stabilize mucin entanglements or even link mucin oligomers alongside most of their contour lengths (i.e., through a zipper-like mechanism). If so, the asymmetry of the mucin macromolecules would lead to the formation of anisotropic networks (27). In addition, $\mathrm{Ca}^{2+}$ could bind other glycoproteins and anionic proteins to the nascent mucin matrix. Second, the increasingly lower $\mathrm{pH}$ and higher $\left[\mathrm{Ca}^{2+}\right]$ might favor interchain links involving one or more of the cysteine-rich protein domains found in gel-forming mucins and other matrix proteins. In support of these two notions, several studies have shown that gel-forming mucins aggregate and/or self-oligomerize through protein-rich regions, O-glycans, or both in response to increased $\left[\mathrm{Ca}^{2+}\right]$ and/or $\left[\mathrm{H}^{+}\right](14-16,28-30)$. Third, a higher intraluminal $\left[\mathrm{H}^{+}\right]$and $\left[\mathrm{Ca}^{2+}\right]$ is expected to diminish the repulsive forces among the negatively charged mucin oligomers, in- creasing the chances for forming more entanglements as mucins oligomerize (i.e., the ratio between $L$ and $V$ decreases). Therefore, it seems reasonable to assume that mucins in the transGolgi compartments and beyond are forming a polyanionic cross-linked network, which eventually will become the granule mucin matrix. By definition, a network of cross-linked polymers in a liquid medium is a gel, and importantly the volume of gels can be modulated by small changes in the environment, a property that might explain mucin intragranular condensation (14).

\section{GEL-FORMING MUCINS: INTRAGRANULAR ORGANIZATION}

\section{Gel Volume Transitions and Intragranular Mucins}

Studies with synthetic polyanionic gels have shown that under certain conditions gels are able to undergo a discontinuous volume (phase) transition between a swollen and a collapsed phase $(31,32)$. Gel volume transitions have three major properties: $(i)$ they can result from small changes in the external conditions that affect the gel, including temperature, solvent, $\mathrm{pH}$, ionic composition, and so on; (ii) the changes in gel volume can be massive (up to 1,000-fold); and (iii) they are reversible (i.e., a collapsed gel can abruptly swell on small changes in the environment). In polyanionic gels, the balance among three competing forces determines the (transition) critical point (32): $(i)$ the positive osmotic pressure of counter ions, which tends to swell the gel; (ii) the negative pressure due to polymer-polymer affinity, which tends to shrink the gel; and (iii) the rubber elasticity of the polymer network, which makes the gel to expand (contract) whenever the gel is contracted (expanded). All these forces vary with temperature, $\mathrm{pH}$, salts, and so forth. It is important to mention here, however, that phase transitions are not necessarily rapid or sudden (32). Further, the same gel can contract or expand by a continuous, rather than a discontinuous, process just by changing the nature of the fluid in which it is embedded. Though these studies have a solid mathematical and experimental basis, which anticipates the existence of a phase transition critical point irrespective of the gel nature, the question arises whether such critical points can be reached within dynamic cellular compartments like those involved in protein secretion.

In principle, there are five major reasons to suggest that a volume transition takes place during mucin granule biogenesis. First, as discussed above, intralumenal mucin oligomers in the late Golgi compartments and beyond probably form a network by topological entanglements and noncovalent links. Second, increased $\left[\mathrm{H}^{+}\right]$and $\left[\mathrm{Ca}^{2+}\right]$ in these cellular compartments (14) could be the triggering factors for the volume transition by increasing the affinity between mucin strands. Moreover, these luminal changes occur at the right intracellular site, that is, where the polyanionic nature of mucins is acquired. Third, a volume transition mechanism would allow reaching a high degree of intragranular condensation. Fourth, the biogenesis of the granule is a continuous but slow process that takes more than $12 \mathrm{~h}$ to be completed (J. Perez-Vilar, unpublished observations) and, therefore, there is a large period of time during which optimal conditions for a volume transition could develop. Fifth, granule exocytosis involves the expansion of the mucin matrix (14), and because volume transitions are reversible (32), a single mechanism would explain both the condensation and decondensation of the granule matrix.

\section{The Matrix Core Paradigm}

The current paradigm explaining mucin granule organization and biogenesis was originally proposed by the Verdugo laboratory on the basis of video-enhanced microscopy studies that made it 
possible to visualize and monitor the discharge of mucin granules in cultured mucous cells $(14,33-36)$. Thus, upon fusion of the granule with the plasma membrane, the intragranular (mucin) matrix abruptly swelled, discharging the accompanying secretory products. Matrix swelling had three major features: $(i)$ it was massive (i.e., the matrix increased its volume many times); (ii) it had a kinetic similar to the swelling of condensed artificial polymer gels, including a high Hill coefficient; and (iii) it could be arrested to some extent by increasing the extracellular concentrations of $\mathrm{Ca}^{2+}$ and $\mathrm{H}^{+}$, suggesting that these two cations were critical for the mechanism of mucin intragranular condensation. Indeed, it has been shown that the mucin granule lumen is rich in $\mathrm{Ca}^{2+}$ and $\mathrm{H}^{+}(37,38)$ In view of these properties, and similar observations in other regulated secretory cell systems (39-41), it is suggested that: $(i)$ intragranular polyanionic matrices are condensed; ( $i$ i) high concentrations of $\mathrm{Ca}^{2+}$ and $\mathrm{H}^{+}$contribute to matrix condensation by neutralizing (shielding) mucin negative charges and stabilizing chain entanglements; and (iii) matrix swelling (decondensation) involves a phase transition process similar to the phase transition of condensed chemical polymer gels (see above). Further studies suggest that upon activation by proper signals, the bound $\mathrm{Ca}^{2+}$ is displaced by incoming $\mathrm{K}^{+}(39$, $40,42,43)$ or $\mathrm{Cl}^{-}$(37). Disruption of $\mathrm{Ca}^{2+}$ shielding would result in a rapid increase of repulsive forces among mucin sialic and sulfate groups, and eventually the decondensation and hydration of the granule matrix $(14,37)$.

Similarly, a mucin phase transition-based mechanism could be envisioned during mucin granule biogenesis (14). Thus, at some point during their transport through the Golgi complex and/or beyond, mucins aggregate, forming a condensed, polyanionic granular matrix. It is suggested that the mucin matrix core: (i) comprises a highly entangled anisotropic network crosslinked by $\mathrm{Ca}^{2+}$; (ii) is immobile and hence able to constrain the movement of secretory proteins, which allows the intragranular accumulation of large amounts of osmotically inert proteins (14, 33,34 ); (iii) is mostly dehydrated (i.e., water molecules just form a hydration shell that surrounds the mucin strands); and (iv) is enclosed by a peripheral fluid phase containing free $\mathrm{Ca}^{2+}$ and other compounds in equilibrium with the outer region of the matrix core $(40,41)$ (Figure 2). This mobile pool of $\mathrm{Ca}^{2+}$ can be

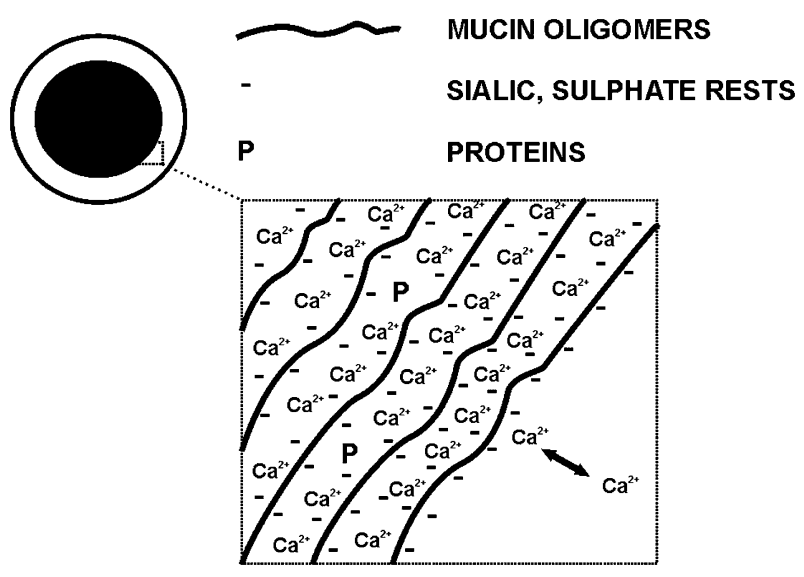

Figure 2. The matrix core model. This model suggests a zipper-like mucin matrix condensation in which $\mathrm{Ca}^{2+}$ ions bind negatively charged mucin oligomers (black lines), filling, together with a hydration shell, most of the space between them. Secretory proteins $(P)$ are entrapped among the condensed matrix. The condensed core is encircled by a fluid aqueous phase in which free $\mathrm{Ca}^{2+}$ and other ions are in equilibrium with their immobile counterpart in the periphery of the matrix core. released from the granule by diffusion during signal transduction $(42,43)$. Consistent with this notion, electro-dense cores of variable sizes enclosed by a peripheral electro-lucent area can be seen by electron microscopy in different regulated secretory granules, including the mucin granule (25). In contrast, it has not been shown that purified mucins form a collapsed network when incubated with $\mathrm{Ca}^{2+}$ at low $\mathrm{pH}$, which suggests that the mechanism of intragranular mucin assembly is more complex than a mere phase transition. In this respect, the existence of intragranular lipids that would increase the affinity between mucin strands has been postulated (14).

\section{The Matrix Meshwork Paradigm}

The notion that the mucin granule lumen comprises a condensed, nondiffusible mucin core was directly tested by performing fluorescence recovery after photobleaching (FRAP) studies on mucin granules of HT29-18N2 mucous/goblet cells stably expressing a fusion protein between green fluorescent protein (GFP) and the CK-domain of MUC5AC that was accumulated in the granule (Figure 3A) (44). The cell line HT29-18N2 is a subclone of the human colonic cancer cell line HT29 that differentiates to mucous/goblet cells (45). Because the protein tracer was not covalently bound to the endogenous mucins (44), it could be used as a soluble FRAP probe to obtain information regarding the mucin granule intraluminal environment (46) (Figure 3B). Whether the granule lumen is altered by the presence of this protein cannot be totally disregarded, although the mucin secretion rate in these and the parental cells are similar, suggesting that this is not the case (J. Perez-Vilar, unpublished observations). In the FRAP studies, large mucin granules (diameters $\geqslant$ $1.1 \mu \mathrm{m}$ ) in live cells were visualized using a laser scanning confocal microscope. A small area in the granule equatorial plane was bleached at high laser power and the recovery of the fluorescence (i.e., the diffusion of the non-bleached molecules inside the bleached area) followed over time. The intragranular FRAP profiles obtained in most granules showed that intraluminal GFP-mucin: ( $i$ ) was fractionated between a mobile and an immobile fraction; and (ii) homogenously, though very slowly $\left(\sim 0.014 \mu \mathrm{m}^{2} \mathrm{~s}^{-1}\right)$, diffused into the bleached area (44) (Figure $3 \mathrm{C})$. These results argued against the existence of a condensed, nondiffusible granule core, the major premise of the matrix core paradigm. As the tracer is a medium-sized compact protein, this suggests that the meshwork minimal average pore size probably is $\geqslant 10 \mathrm{~nm}$. As we have discussed above, it is possible to have that range of pore size in a concentrated mucin solution (i.e., $\left.\phi>\phi^{*}\right)$. Indeed, the average apparent diffusion coefficient of GFP-mucin in the endoplasmic reticulum (47) and the extracellular mucus (J. Perez-Vilar, unpublished observations) were 100fold and $>170$-fold larger than in the mucin granule, consistent with the existence of a partially, rather than totally, condensed granule mucin matrix that is expanded upon granule exocytosis (Figure 4). Whether this matrix is encircled with a fluid in which proteins can move faster, as suggested by the matrix core model, has not yet been investigated.

As expected for a meshwork-like organization, GFP-mucin intragranular diffusion and/or distribution (bound versus mobile fractions) can be altered by inhibitors of post-translational modifications pertinent to mucins (i.e., the granule matrix) (44), including O-glycosylation, sulfation, sialylation, and disulfide bond formation, as well as by inhibiting the activity of the vacuolar $\mathrm{H}^{+}$-ATPase (44). Three major suggestions derived from these studies. First, mucin O-glycans, and specifically the degree of mucin sialylation and sulfation (i.e., charge density), and likely the length of their O-glycans, affect the accessibility of the protein tracer, and possibly other luminal proteins, to the matrix protein-rich regions, and their rate of diffusion through the 
A

a

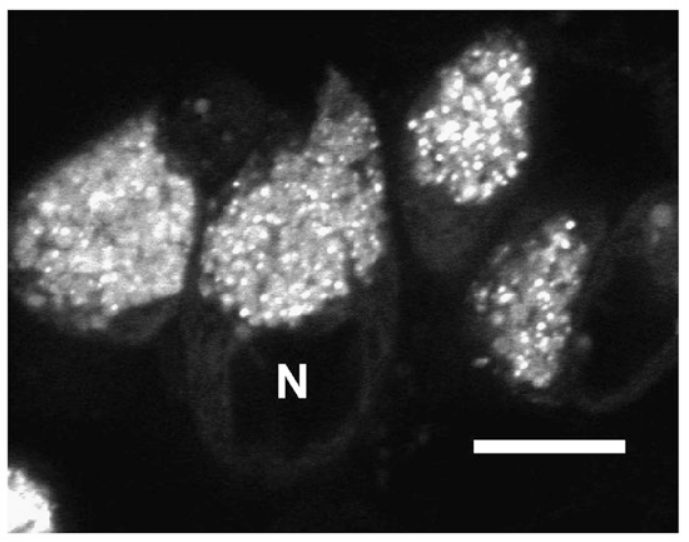

B

a
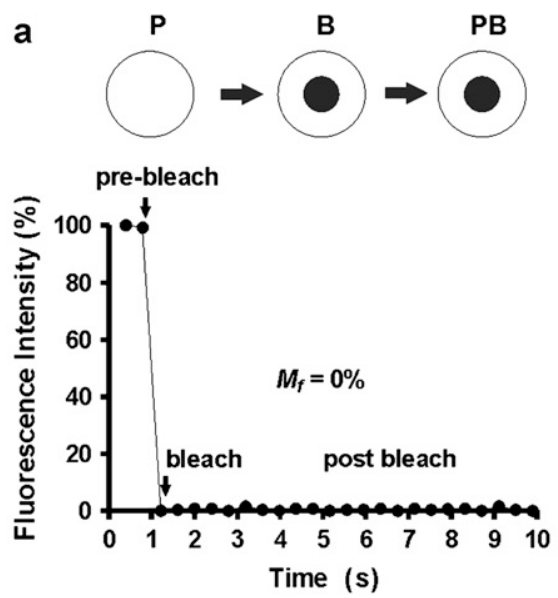

C
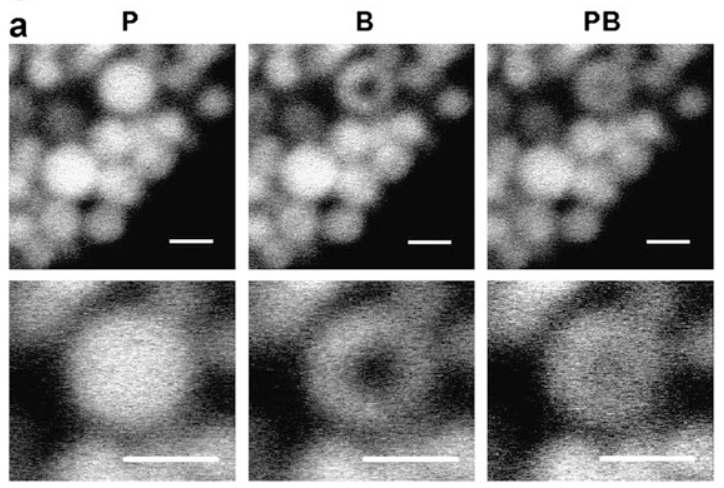

b

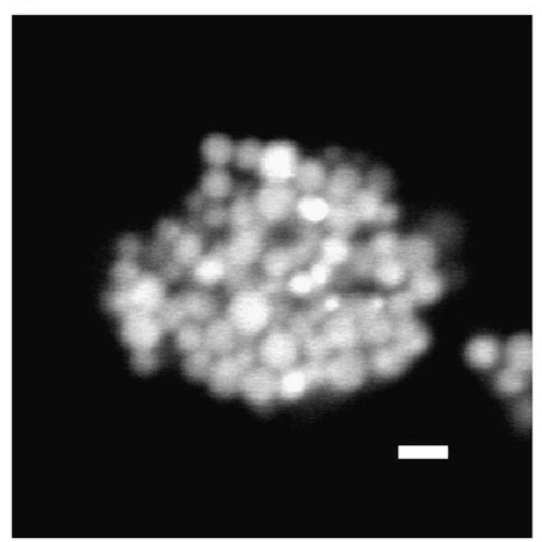

b

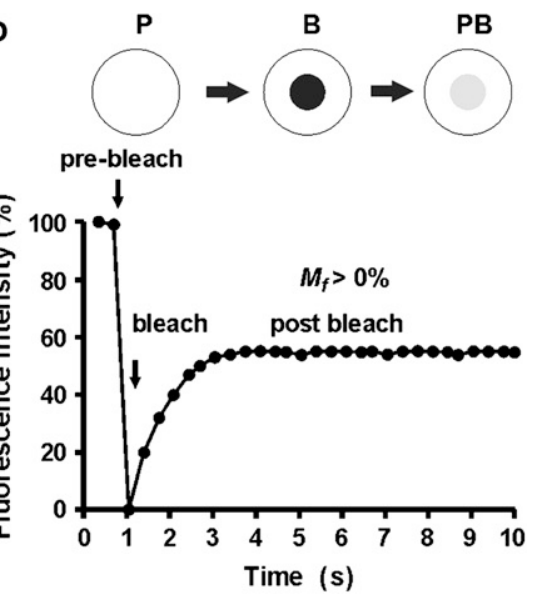

b

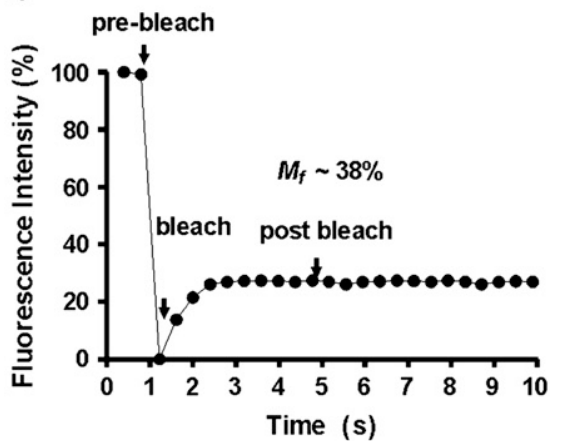

Figure 3. FRAP studies in living mucous/ goblet cells. (A) XY confocal images of HT29-18N2 mucous/goblet cells expressing mucin-GFP showing a cluster of cells $(a)$ or a single cell $(b)$. Note the difference in fluorescence intensities in the granular mass and the rest of the cell, which virtually cannot be seen unless the granule signal is oversaturated. Scale bars $=8 \mu \mathrm{m}$ and $1 \mu \mathrm{m}$ in $a$ and $b$, respectively. (B) Expected normalized FRAP curve profiles for a mucin granule in which the lumen has a condensed matrix core where proteins are unable to diffuse $(a)$ or a penetrable matrix meshwork (panel $b$ ) following the main premise of the matrix core paradigm or the matrix meshwork paradigm, respectively. The matrix core model predicts the absence of fluorescence recovering in the bleached area. Conversely, the matrix meshwork model predicts recovery of the signal assuming that the size and tertiary structure of the protein probe allow for its diffusion through the pores of the meshwork. $\mathrm{P}$, pre-bleach granule; $\mathrm{B}$, granule immediately after bleaching; $P B$, granule once fluorescence recovery reaches a plateau. (C) Representative FRAP analysis involving bleaching of a circular spot of a mucin granule in a living mucous/goblet cell, showing $X Y$ confocal images before, immediately after bleaching, and $5 \mathrm{~s}$ after bleaching, respectively, at two different magnifications (a). Scale bars $=0.5 \mu \mathrm{m}$. Also shown is the actual normalized FRAP bleaching/recovery curve $(b)$. P, pre-bleach image; $B$, granule immediately after bleaching image; $\mathrm{PB}$, image of the granule once fluorescence recovery reaches a plateau. Scale bars $=$ $1 \mu \mathrm{m}$. matrix pores. Second, intraluminal acidic $\mathrm{pH}$ is important to maintain the meshwork frame, and also for the binding of certain secretory proteins to the immobile matrix (44). Hence, conditions leading to altered degree of sialylation and/or sulfation of intragranular mucins, or intraluminal $\mathrm{pH}$, could change to some extent the matrix organization. Third, protein-protein interactions are not absolutely required to maintain the granule organization (47), as judged by the effects of disrupting intragranular disulfide bonds, consistent with the notion that inter-strand links via multivalent ions $\left(\mathrm{Ca}^{2+}\right)$ are sufficient to hold the matrix together at acidic $\mathrm{pH}(14,37)$. It is noteworthy to mention, however, that disulfide reduction usually resulted in a complete immobilization of the tracer in the granule lumen (47). This observation has two, though not necessarily incompatible, explanations: $(i)$ reduction of the protein probe disulfide bonds resulted in its de-folding and subsequent binding to the matrix components; and (ii) the meshwork average pore size drastically diminished after the disulfide reduction. The latter possibility implies a role for protein-protein interactions in keeping a relative large meshwork pore size and ultimately in the organization of the granule matrix. Indeed, protein-protein interactions might have a critical role during granule biogenesis. Thus, when the intragranular $\mathrm{pH}$ was raised, reduction of intragranular disulfide bonds resulted in granule matrix disorganization and partial discharge (47) - that is, the links holding the meshwork at acidic $\mathrm{pH}$ were insufficient as the $\mathrm{pH}$ was raised. These results were 


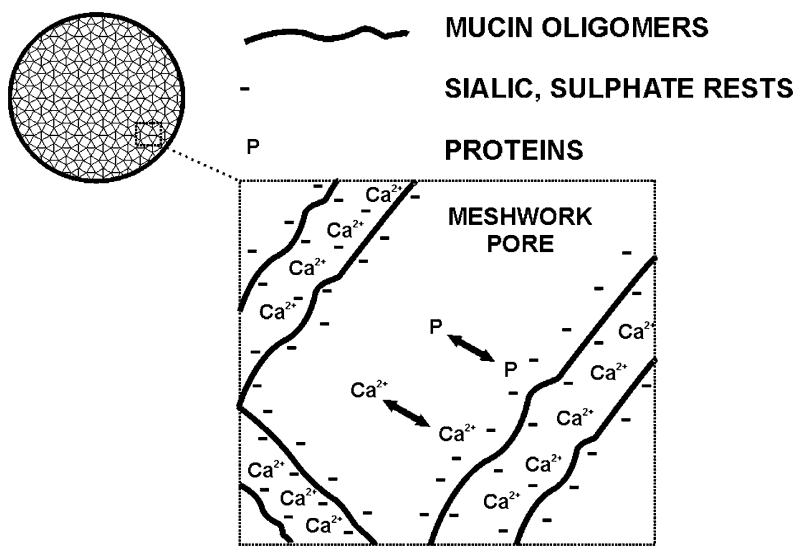

Figure 4. The matrix meshwork model. Our studies suggest the formation of a meshwork in a fluid phase in which proteins $(\mathrm{P})$ and ions $\left(\mathrm{Ca}^{2+}\right)$ can diffuse through its pores and interact with the matrix components, including protein- and O-glycan-rich regions, respectively. $\mathrm{Ca}^{2+}$-dependent condensation might be in effect but would not involve the total collapse of the matrix.

consistent with a role for protein-protein interactions during the early stages of matrix assembly, which takes place in a less acidic environment than the granule lumen. As the granule lumen is increasingly acidified, the $\mathrm{Ca}^{2+}$-dependent inter-strand links are quantitatively more important.

Secretory products in many regulated secretory granules are not free to move (25). They remain immobilized inside a condensed polymer matrix that virtually abolishes their osmotic activity. This scenario is also postulated by the matrix core paradigm for the mucin granule. According to the meshwork model, however, some secretory proteins would be able to very slowly diffuse within the granule lumen and, therefore, an osmotic activity leading to granule steady swelling should be expected. This suggests the existence of an alternative mechanism for securing osmotic equilibrium of the mucin granules.

\section{A Unifying Paradigm?}

The major conceptual difference between both models is in the degree of condensation of the granule matrix. The core paradigm suggests a granule matrix entirely condensed, whereas the meshwork paradigm proposes a partially condensed matrix. Both models anticipate a critical role for intraluminal $\mathrm{Ca}^{2+}$ and $\mathrm{H}^{+}$. It is important to note that the core paradigm was postulated on the basis of studies that tried to explain the granule matrix decondensation upon exocytosis (14). Thus, no direct evidence was provided regarding the volume phase transition that presumably generated the mucin granule matrix. Indeed, it was necessary to postulate the existence of intragranular lipids and/or other hydrophobic compounds to explain mucin condensation (14). Conversely, the studies leading to the matrix meshwork model were designed to investigate the intragranular organization $(44,47)$. Because of the nature of the FRAP protein probe, the mechanism of matrix decondensation was out of the reach of these studies. Hence, a volume transition of a partially condensed granule matrix during exocytosis would be strictly consistent with the experimental data available. Moreover, while a discontinuous volume transition of the granule matrix during exocytosis suits the sudden and drastic changes of conditions that take place as the matrix encounters the extracellular compartment, it is unclear if a similar situation occurs during granule biogenesis. Thus, as gel-forming mucin oligomers traverse the trans-Golgi compartments, immature and mature granules, the luminal environment gradually, rather than suddenly, changes. Under these conditions, long-term (discontinuous or continuous) volume transitions might be taking place. This would account for the persistence of partially condensed matrix meshwork and the absence of cores in most granules.

Interestingly, fluorescence-free, centrally located small cores were observed in a small fraction of the granules of living mucous cells (44). In these granules, the rest of the lumen appeared to be constituted by a permeable meshwork as in most of the granules. If these cores are the counterparts of the cores observed in granules of fixed cells, a total collapse of the matrix meshwork in those small regions likely took place. This observation may suggest that the lumen in these granules, and likely the rest of the granules, is near the (volume transition) critical point. Interestingly, the configuration of synthetic polymer networks changes considerably as conditions come close to the critical point (32). Thus, collapsed regions are regularly inter-dispersed with entirely de-condensed areas, and ultimately forming a network with larger pores than an entirely expanded gel network.

\section{INTRAGRANULAR MUCINS AND CFTR: A FUNCTIONAL LINK?}

The lack of functional cystic fibrosis (CF) transmembrane conductance regulator (CFTR) in airway epithelial cells leads to isotonic absorption of water, which sequentially results in mucus stasis and accumulation, bacterial infection, and lung inflammation $(48,49)$. Although it is increasingly clear that the viscoelastic properties of the mucus are drastically compromised because of lower water content and the presence of other macromolecules such as DNA and actin, the debate on whether CF mucins are intrinsically different still is alive (50). Since the 1970 s to the present, disparate results have been reported regarding differences in CF mucins glycosylation and sulfation, and no widespread consensus has been reached (e.g., Refs. 51, 52, and the references therein). An intrinsic mucin defect (i.e., independent of bacterial infection and lung inflammation) would likely, but not necessarily (see below), mean that CFTR is expressed in goblet/mucous cells. Recent studies not only suggest that CFTR is part of the mucin granule membrane but also that its failure alters granule organization and, ultimately, matrix expansion after exocytosis $(36,37,53)$. The mucin granules in $\mathrm{CF}$ airway glandular cells had less water and higher ion content than in granules of normal cells (53). Similarly, increased $\mathrm{Ca}^{2+}$ concentration was found in the mucin granule lumen of cultured gallbladder epithelial cells from $C f t r^{(-/-)}$mice, while secreted mucins were less sulfated than their respective normal counterparts (36). In both cell systems, granule expansion upon exocytosis was abnormal, which suggests that intragranular organization is altered in CF mucous cells. Interestingly, the intragranular mobility of mucin-GFP in HT29 cells increased on protein sulfation inhibition (47), suggesting that the charge density altered the diffusion of the protein or that the pore size of the meshwork increased. Although other investigators have failed to detect CFTR in mucous/goblet cells (e.g., Ref. 54), the expression of this channel would not be strictly required to alter intracellular mucin glycosylation/sulfation. Because competition among different sulfo-, sialyl-, and fucosyltransferases is critical for the control of $\mathrm{O}$-glycosylation elongation and termination reactions $(55,56)$, the biosynthesis of mucin-type O-glycans in a given cell depends on the specific glycosyltransferses/sulfotransferases expressed, and their levels of expression. Hence, it can be speculated that indirect de-regulation of the expression of one or more glycosyltransferases and/or sulfotransferases would suffice for the synthesis of abnormal mucins (57). 


\section{SUMMARY AND FUTURE DIRECTIONS}

The studies in live mucous/goblet cells suggest the granule matrix would be embedded in a fluid in which secretory products very slowly diffuse, and interact with the matrix meshwork. Mucin O-glycans and their anionic (sulfate and sialic) groups would: (i) prevent the access to protein regions in the matrix, (ii) affect protein diffusion in the fluid by steric and charge hindrances, and (iii) establish intermolecular interactions among mucins oligomers/multimers through ionic $\left(\mathrm{Ca}^{2+}\right)$ bonds. Other luminal proteins, especially sulfated proteoglycans, could also control protein diffusion through the matrix pores (47).

Among the many questions that should focus future research efforts are: What kind of luminal events take place before granule formation? How is the matrix meshwork frame organized? What sorts of interactions happen between mucins before or during matrix assembly? What proteins, besides mucins, are required for matrix assembly? Does the mucin granule mature and what does this mean in the context of matrix structure? Is matrix assembly coupled with recruitment of other granule (membrane and soluble) proteins? What is the nature of the mechanism that maintains the granule osmotic equilibrium? and so on.

Conflict of Interest Statement: J.P.-V. does not have a financial relationship with a commercial entity that has an interest in the subject of this manuscript.

Acknowledgments: The author thanks Drs. P. Verdugo (Department of Bioengineering, University of Washington, WA) and T. Carter (Division of Molecular Neuroendocrinology, National Institute for Medical Research, UK) for critically reading the manuscript.

\section{References}

1. Perez-Vilar J, Hill RL. The structure and assembly of secreted mucins. J Biol Chem 1999;274:31751-31754.

2. Dekker J, Rossen JW, Buller HA, Einerhand AW. The MUC family: an obituary. Trends Biochem Sci 2002;27:126-131.

3. Sheehan JK, Brazeau C, Kutay S, Pigeon H, Kirkham S, Howard M, Thornton DJ. Physical Characterization of the MUC5AC mucin: a highly oligomeric glycoprotein whether isolated from cell culture or in vivo from respiratory mucous secretions. Biochem J 2000;347:37-44.

4. Fowler J, Vinall L, Swallow D. Polymorphism of the human muc genes. Front Biosci 2001;6:D1207-D1215.

5. Perez-Vilar J, Randell SH, Boucher RC. C-Mannosylation of MUC5AC and MUC5B Cys subdomains. Glycobiology 2004;4:325-337.

6. Perez-Vilar J, Eckhardt AE, DeLuca A, Hill RL. Porcine submaxillary mucin forms disulfide-linked multimers through its amino-terminal D-domains. J Biol Chem 1998;273:1442-1449.

7. Lidell ME, Johansson ME, Hansson GC. An autocatalytic cleavage in the $\mathrm{C}$ terminus of the human MUC2 mucin occurs at the low $\mathrm{pH}$ of the late secretory pathway. J Biol Chem 2003;278:13944-13955.

8. Forstner G. Signal transduction, packaging and secretion of mucins. Annu Rev Physiol 1995;57:585-605.

9. Li Y, Martin LD, Spizz G, Adler KB. MARCKS protein is a key molecule regulating mucin secretion by human airway epithelial cells in vitro. J Biol Chem 2001;276:40982-40990.

10. Rogers DF. The airway goblet cell. Int J Biochem Cell Biol 2003;35:1-6.

11. Velcich A, Yang W, Heyer J, Fragale A, Nicholas C, Viani S, Kucherlapati R, Lipkin M, Yang K, Augenlicht L. Colorectal cancer in mice genetically deficient in the mucin Muc2. Science 2002;295:1726-1729.

12. Doi M, See H. Introduction to polymer physics. Oxford: Oxford University Press; 1995.

13. Graessley WW. Polymeric liquids and networks: structure and properties. New York and London: Garland Sciences; 2004.

14. Verdugo P. Goblet cells secretion and mucogenesis. Annu Rev Physiol 1990;52:157-176.

15. Bansil R, Stanley E, LaMont JT. Mucin biophysics. Annu Rev Physiol 1995;57:635-657.

16. Bansil R, Turner BS. Mucin structure, aggregation, physiological functions and biomedical applications. Curr Opin Colloid Interf Sci 2006;11: $164-170$.
17. Raynal BD, Hardingham TE, Thornton DJ, Sheehan JK. Concentrated solutions of salivary MUC5B mucin do not replicate the gel-forming properties of saliva. Biochem J 2002;362:289-296.

18. Taylor C, Allen A, Dettmar PW, Pearson JP. The gel matrix of gastric mucus is maintained by a complex interplay of transient and nontransient associations. Biomacromolecules 2003;4:922-927.

19. De Gennes PG. Scalling concepts in polymer physics. New York: Cornell University Press; 1979.

20. Strous GJ, Dekker J. Mucin-type glycoproteins. Crit Rev Biochem Mol Biol 1992;27:57-92.

21. Shogren R, Gerken TA, Jentoft N. Role of glycosylation on the conformation and chain dimensions of O-linked glycoproteins: light-scattering studies of ovine submaxillary mucin. Biochemistry 1989;28:5525-5536.

22. Gerken TA. Biophysical approaches to salivary mucin structure, conformation and dynamics. Crit Rev Oral Biol Med 1993;4:261-270.

23. Deschuyteneer M, Eckhardt AE, Roth J, Hill RL. The subcellular localization of apomucin and nonreducing terminal $\mathrm{N}$-acetylgalactosamine in porcine submaxillary glands. J Biol Chem 1988;263:2452-2459.

24. Thiele C, Huttner WB. Protein and lipid sorting from the trans-Golgi network to secretory granules: recent developments. Semin Cell Dev Biol 1998;9:511-516.

25. Burgoyne RD, Morgan A. Secretory granule exocytosis. Physiol Rev 2003;83:581-632.

26. Verdugo P, Deyrup-Olsen I, Aitken M, Villalon M, Johnson D. Molecular mechanism of mucin secretion: I. The role of intragranular charge shielding. J Dent Res 1987;66:506-508.

27. Viney C, Huber AE, Verdugo P. Liquid crystalline order in mucus. Macromolecules 1993;26:852-855.

28. McCullagh CM, Jamieson AM, Blackwell J, Gupta R. Viscoelastic properties of human tracheobronchial mucin in aqueous solution. Biopolymers 1995;35:149-159.

29. Cao X, Bansil R, Bhaskar KR, Turner BS, LaMont JT, Niu N, Afdhal NH. pH-dependent conformational change of gastric mucin leads to sol-gel transition. Biophys J 1999;76:1250-1258.

30. Raynal BD, Hardingham TE, Sheehan JK, Thornton DJ. Calciumdependent protein interactions in MUC5B provide reversible crosslinks in salivary mucus. $J$ Biol Chem 2003;278:28703-28710.

31. Tanaka T. Collapse of gels and the critical end point. Phys Rev Lett 1978;40:820-823.

32. Li Y, Tanaka T. Phase transitions of gels. Annu Rev Mater Sci 1992; 22:243-277.

33. Verdugo P. Goblet cell Hydration kinetics of exocytosed mucins in cultured secretory cells of the rabbit trachea: a new model. Ciba Found Symp 1984;109:212-225.

34. Verdugo P. Mucin exocytosis. Am Rev Respir Dis 1991;144:S33-S37.

35. Espinosa M, Noe G, Troncoso C, Ho SB, Villalon M. Acidic pH and increasing $\left[\mathrm{Ca}^{2+}\right]$ reduce the swelling of mucins in primary cultures of human cervical cells. Hum Reprod 2002;17:1964-1972.

36. Kuver R, Wong T, Klinkspoor JH, Lee SP. Absence of CFTR is associated with pleitropic effects on mucins in mouse gallbladder epithelial cells. Am J Physiol Gastrointest Liver Physiol 2006;291:G1148-G1154.

37. Kuver R, Klinkspoor JH, Osborne WRA, Lee SP. Mucous granule exocytosis and CFTR expression in gallbladder epithelium. Glycobiology 2000;10:149-157.

38. Chin WC, Quesada I, Nguyen T, Verdugo P. Oscillations of pH inside the secretory granule control the gain of $\mathrm{Ca} 2+$ release for signal transduction in goblet cell exocytosis. Novartis Found Symp 2002;248: 132-141.

39. Marszalek PE, Farrell B, Verdugo P, Fernandez JM. Kinetics of realase of serotonin from isolated secretory granules. I. Amperometric detection of serotonin from electroporated granules. Biophys J 1997;73: 1160-1168.

40. Marszalek PE, Farrell B, Verdugo P, Fernandez JM. Kinetics of realase of serotonin from isolated secretory granules. II. Ion exchange determines the diffusivity of serotonin. Biophys $J$ 1997;73:1169-1183.

41. Chin WC, Orellana MV, Quesada I, Verdugo P. Secretion in unicellular marine phytoplankton: demonstration of regulated exocytosis in Phaeocystis globosa. Plant Cell Physiol 2004;45:535-542.

42. Nguyen T, Chin WC, Verdugo P. Role of $\mathrm{Ca} 2+/ \mathrm{K}+$ ion exchange in intracellular storage and realase of mucin. Nature 1998;395:908-912.

43. Quesada I, Chin WC, Verdugo P. ATP-independent luminal oscillations and realase of $\mathrm{Ca} 2+$ and $\mathrm{H}+$ from mast cell secretory granules: implications for signal transduction. Biophys J 2003;85:963-970.

44. Perez-Vilar J, Olsen JC, Chua M, Boucher RC. pH-dependent intraluminal organization of mucin granules in live human mucous/goblet cells. J Biol Chem 2005;280:16868-16881. 
45. Phillips TE, Huet C, Bilbo PR, Podolsky DK, Louvard D, Neutra MR. Human intestinal goblet cells in monolayer culture: characterization of a mucus-secreting subclone derived from the HT29 colon adenocarcinoma cell line. Gastroenterology 1988;94:1390-1403.

46. Lippincott-Schwartz J, Patterson GH. Development and use of fluorescent protein markers in living cells. Science 2003;300:91-96.

47. Perez-Vilar J, Mabolo R, McVaugh CT, Bertozzi CR, Boucher RC. Mucin granule intraluminal organization in living mucous/goblet cells. Roles of protein post-translational modifications and secretion. J Biol Chem 2006;281:4844-4855.

48. Boucher RC. An overview of the pathogenesis of cystic fibrosis lung disease. Adv Drug Deliv Rev 2002;54:1359-1371.

49. Rose MC, Voynow JA. Respiratory tract mucin genes and mucin glycoproteins in health and disease. Physiol Rev 2006;86:245-278.

50. Perez-Vilar J, Boucher RC. Re-evaluating gel-forming mucins' roles in cystic fibrosis lung disease. Free Radic Biol Med 2004;37:1564-1577.

51. Schulz BL, Sloane AJ, Robinson LJ, Sebastian LT, Glanville AR, Song Y, Verkman AS, Harry JL, Packer NH, Karlsson NG. Mucin glycosylation changes in cystic fibrosis lung disease are not manifest in submucosal gland secretions. Biochem J 2005;387:911-919.

52. Xia B, Royall JA, Damera G, Sachdev GP, Cummings RD. Altered
O-glycosylation and sulfation of airway mucins associated with cystic fibrosis. Glycobiology 2005;15:747-775.

53. Baconnais S, Delavoie F, Zahm JM, Milliot M, Terryn C, Castillon N, Banchet V, Michel J, Danos O, Merten M, et al. Abnormal ion content, hydration and granule expansion of the secretory granules from cystic fibrosis airways glandular cells. Exp Cell Res 2005;309:296-304.

54. Abdullah LH, Conway JD, Cohn JA, Davis CW. Protein kinase C and $\mathrm{Ca}^{2+}$ activation of mucin secretion in airway goblet cells. Am J Physiol 1997;273:L201-L210.

55. Brockhausen I. Sulphotransferases acting on mucin-type oligosaccharides. Biochem Soc Trans 2003;31:318-325.

56. Hanish FG. O-glycosylation of the mucin type. Biol Chem 2001;382:143149.

57. Roussel P, Lamblin G. The glycosylation of airway mucins in cystic fibrosis and its relationship with lung infection by Pseudomonas aeruginosa. Adv Exp Med Biol 2003;535:17-32.

58. Buisine MP, Desseyn JL, Porchet N, Degand P, Laine A, Aubert JP. Genomic organization of the 3'-region of the human MUC5AC mucin gene: additional evidence for a common ancestral gene for the $11 \mathrm{p} 15.5$ mucin gene family. Biochem J 1998;332:729-738.

59. Escande F, Aubert JP, Porchet N, Buisine MP. Human mucin gene MUC5AC: organization of its 5'-region and central repetitive region. Biochem J 2001;358:763-772. 